\title{
Inverse Problem for Interior Spectral Data of the Dirac Operator on a Finite Interval
}

\author{
By
}

Kiyoshi Mochizuki* and Igor Trooshin**

\begin{abstract}
In this paper the inverse problems for the Dirac operator on a finite interval are studied. A set of values of eigenfunctions in some internal point and spectrum are taken as a data. Uniqueness theorems are obtained. The approach that was used in the investigation of inverse problems for interior spectral data of the Sturm-Liouville operator is employed.
\end{abstract}

\section{$\S 1 . \quad$ Introduction}

We consider the canonical form of the Dirac operator $L$ (see [6]), generated by the differential expression

$$
l(y)=B y^{\prime}+Q(x) y \quad(0 \leq x \leq 1)
$$

with

$$
B=\left(\begin{array}{cc}
0 & 1 \\
-1 & 0
\end{array}\right), \quad Q(x)=\left(\begin{array}{cc}
p(x) & q(x) \\
q(x) & -p(x)
\end{array}\right), \quad y(x)=\left(\begin{array}{l}
y_{1}(x) \\
y_{2}(x)
\end{array}\right)
$$

subject to the boundary conditions

$$
\begin{array}{ll}
y_{1}(0) \cos \alpha+y_{2}(0) \sin \alpha=0, & 0 \leq \alpha<\pi, \\
y_{1}(1) \cos \beta+y_{2}(1) \sin \beta=0, & 0 \leq \beta<\pi .
\end{array}
$$

Communicated by T. Kawai. Received May 9, 2001. Revised December 20, 2001.

2000 Mathematics Subject Classification(s): 34L05, 65L09.

* Department of Mathematics, Tokyo Metropolitan University, Hachioji, Tokyo 192-0397, Japan.

Current address: Department of Mathematics, Faculty of Science and Engineering, Chuo University, Bunkyo, Tokyo 112-8551, Japan.

e-mail: mochizuk@math.chuo-u.ac.jp

** Precision Mechanics and Control Institute of Russian Academy of Sciences, 24, Rabochaya, Saratov 410028, Russia. 
Throughout this paper we require that $p(x), q(x) \in C^{1}[0,1]$ and are real-valued functions. This regularity assumption is too much restrictive. It seems to be sufficient only to require that $p(x), q(x) \in L^{2}(0.1)$. In this paper, however, we do not enter into this problem.

As is well known, the operator $L$ has a discrete spectrum consisting of simple eigenvalues $\lambda_{n}, n \in Z$. We denote by $y_{n}(x)=\left(y_{n, 1}(x), y_{n, 2}(x)\right)^{T}, n \in$ $Z$, the corresponding eigenfunctions.

Research of inverse problems for Dirac operator follows investigations of closely related inverse problems for Sturm-Liouville operator. T. N. Arutyunyan [1] obtained an analog of Marchenko theorem [8], [9]: he proved that the eigenvalues $\lambda_{n}, n=0, \pm 1, \pm 2, \ldots$ and normalising coefficients $\alpha_{n}=$ $\left\|y_{n}\right\|_{\left\{L^{2}(0.1)\right\}^{2}}, \quad n=0, \pm 1, \pm 2, \ldots \quad\left(\right.$ for $\left.y_{n, 1}(0)=\sin \alpha, y_{n, 2}(0)=-\cos \alpha\right)$ uniquely determined the potential $Q(x)$. M. M. Malamud [7] proved an ana$\log$ of Borg theorem [2]: he showed that the spectra of two boundary value problems for an operator with different boundary conditions at one end (and identical conditions repeated at the other end) uniquely determined the potential $Q(x)$. He also proved an analog of the theorem of Hochstadt and Lieberman [4]: one spectrum and a potential on the interval $(0,1 / 2)$ uniquely determined the potential $Q(x)$ on the whole interval $[0,1]$.

The aim of our present work is to investigate a possibility to recover potential from the known eigenvalues and some information on eigenfunctions in the internal point $b \in(0,1)$. The similar problem for the Sturm-Liouville operator is formulated and studied in our papers [10], [11]. In these articles we have employed technique similar to those used in [4], [12].

\section{$\S 2 . \quad$ Statement of Results}

Let us introduce a second Dirac operator $\tilde{L}$ generated by the differential expression

$$
l(y)=B y^{\prime}+\tilde{Q}(x) y \quad(0 \leq x \leq 1),
$$

subject to the same boundary conditions (2), (3). Here

$$
\tilde{Q}(x)=\left(\begin{array}{cc}
\tilde{p}(x) & \tilde{q}(x) \\
\tilde{q}(x) & -\tilde{p}(x)
\end{array}\right)
$$

with a real-valued functions $\tilde{p}(x), \tilde{q}(x) \in C^{1}[0,1]$. The eigenvalues and the corresponding eigenfunctions of $\tilde{L}$ are denoted by $\tilde{\lambda}_{n}$ and $\tilde{y}_{n}(x)=\left(\tilde{y}_{n, 1}(x), \tilde{y}_{n, 2}\right.$ $\times(x))^{T}(n \in Z)$, respectively. 
Theorem 2.1. If for any $n \in Z$

$$
\lambda_{n}=\tilde{\lambda}_{n}, \quad \frac{y_{n, 1}\left(\frac{1}{2}\right)}{y_{n, 2}\left(\frac{1}{2}\right)}=\frac{\tilde{y}_{n, 1}\left(\frac{1}{2}\right)}{\tilde{y}_{n, 2}\left(\frac{1}{2}\right)},
$$

then $p(x)=\tilde{p}(x), q(x)=\tilde{q}(x)$ on the $[0,1]$.

In the case $b \neq 1 / 2$, the uniqueness of $Q(x)$ can be proven if we require the knowledge of part of the second spectrum.

We denote by $\mu_{n}$ and $\tilde{\mu}_{n}$ respectivly the eigenvalues of problems (1) and (4) subject to the same boundary conditions (2) and

$$
y_{1}(1) \cos \gamma+y_{2}(1) \sin \gamma=0, \quad 0 \leq \gamma<\pi, \quad \gamma \neq \beta
$$

Let $l(n), r(n)$ be sequences of natural numbers with property

$$
\begin{gathered}
l(n)=\frac{n}{\sigma_{1}}\left(1+\epsilon_{1, n}\right), \quad 0<\sigma_{1} \leq 1, \quad \epsilon_{1, n} \rightarrow 0, \\
r(n)=\frac{n}{\sigma_{2}}\left(1+\epsilon_{2, n}\right), \quad 0<\sigma_{2} \leq 1, \quad \epsilon_{2, n} \rightarrow 0 .
\end{gathered}
$$

Theorem 2.2. Let $l(n), r(n)$ and $1 / 2<b<1$ are so chosen that $\sigma_{1}>2 b-1, \sigma_{2}>2-2 b$. If for any $n \in Z$

$$
\lambda_{n}=\tilde{\lambda}_{n}, \quad \mu_{l(n)}=\tilde{\mu}_{l(n)}, \quad \frac{y_{r(n), 1}(b)}{y_{r(n), 2}(b)}=\frac{\tilde{y}_{r(n), 1}(b)}{\tilde{y}_{r(n), 2}(b)},
$$

then $p(x)=\tilde{p}(x), q(x)=\tilde{q}(x)$ on the $[0,1]$.

\section{$\S 3 . \quad$ Proof of Theorem 2.1}

We shall first mention some results which will be needed later.

Let us denote by $w(x, \lambda)=\left(w_{1}(x, \lambda), w_{2}(x, \lambda)\right)^{T}$ and $\tilde{w}(x, \lambda)=\left(\tilde{w}_{1}(x, \lambda)\right.$, $\left.\tilde{w}_{2}(x, \lambda)\right)^{T}$ the solutions of initial-value problems

$$
\begin{gathered}
B w^{\prime}+Q(x) w=\lambda w, \\
w_{1}(0)=\sin \alpha, \quad w_{2}(0)=-\cos \alpha
\end{gathered}
$$

and

$$
\begin{gathered}
B \tilde{w}^{\prime}+\tilde{Q}(x) \tilde{w}=\lambda \tilde{w} \\
\tilde{w}_{1}(0)=\sin \alpha, \quad \tilde{w}_{2}(0)=-\cos \alpha .
\end{gathered}
$$


There exist (see [3], [6]) kernels $K(x, t)=\left(K_{i j}(x, t)\right)_{i, j=1}^{2}, \tilde{K}(x, t)=\left(\tilde{K}_{i j}\right.$ $\times(x, t))_{i, j=1}^{2}$, with entries continuously differentiable on $0 \leq t \leq x \leq 1$ such that

$$
\begin{aligned}
& w(x, \lambda)=\phi_{0}(x, \lambda)+\int_{0}^{x} K(x, t) \phi_{0}(t, \lambda) d t \\
& \tilde{w}(x, \lambda)=\phi_{0}(x, \lambda)+\int_{0}^{x} \tilde{K}(x, t) \phi_{0}(t, \lambda) d t .
\end{aligned}
$$

Here $\phi_{0}(x, \lambda)=(\sin (\lambda x+\alpha),-\cos (\lambda x+\alpha))^{T}$.

Proof of Theorem 2.1. If we multiply (4) (in the sense of scalar product in $\left.R^{2}\right)$ by $\tilde{w}(x, \lambda)$ and the equation of $\tilde{w}(x, \lambda)$ by $w(x, \lambda)$ and subtract, after integrating from 0 to $1 / 2$, we obtain

$$
\begin{aligned}
& \int_{0}^{\frac{1}{2}}\langle(Q(x)-\tilde{Q}(x)) w(x, \lambda), \tilde{w}(x, \lambda)\rangle d x \\
& \quad=\left.\left(\tilde{w}_{2}(x, \lambda) w_{1}(x, \lambda)-\tilde{w}_{1}(x, \lambda) w_{2}(x, \lambda)\right)\right|_{0} ^{\frac{1}{2}} .
\end{aligned}
$$

Since $w(x, \lambda)$ and $\tilde{w}(x, \lambda)$ satisfy the same initial conditions, it follows that

$$
\tilde{w}_{2}(0, \lambda) w_{1}(0, \lambda)-\tilde{w}_{1}(0, \lambda) w_{2}(0, \lambda)=0 .
$$

Define

$$
P(x)=Q(x)-\tilde{Q}(x), \quad p_{1}(x)=p(x)-\tilde{p}(x), \quad q_{1}(x)=q(x)-\tilde{q}(x)
$$

and

$$
H(\lambda)=\int_{0}^{\frac{1}{2}}\langle P(x) w(x, \lambda), \tilde{w}(x, \lambda)\rangle d x .
$$

Then from the conditions of the theorem it follows that

$$
\tilde{w}_{2}\left(\frac{1}{2}, \lambda_{n}\right) w_{1}\left(\frac{1}{2}, \lambda_{n}\right)-\tilde{w}_{1}\left(\frac{1}{2}, \lambda_{n}\right) w_{2}\left(\frac{1}{2}, \lambda_{n}\right)=0
$$

and hence

$$
H\left(\lambda_{n}\right)=0, \quad n \in Z
$$

We can show from $(6),(7)$ that

(8) $H(\lambda)=\int_{0}^{\frac{1}{2}} p_{1}(x)\left[-\cos 2(\lambda x+\alpha)+\int_{0}^{x} R_{1}(x, t) \exp (2 i \lambda t) d t\right.$

$$
\begin{aligned}
& \left.+\int_{0}^{x} R_{2}(x, t) \exp (-2 i \lambda t) d t\right] d x+\int_{0}^{\frac{1}{2}} q_{1}(x)[-\sin 2(\lambda x+\alpha) \\
& \left.+\int_{0}^{x} R_{3}(x, t) \exp (2 i \lambda t) d t+\int_{0}^{x} R_{4}(x, t) \exp (-2 i \lambda t) d t\right] d x
\end{aligned}
$$


where $R_{l}(x, t), l=1, \ldots, 4$, are piecewise-continuously differentiable on $0 \leq$ $t \leq x \leq 1$.

Therefore it follows that $H(\lambda)$ is an entire function of order no greater than 1.

We next define the function

$$
\omega(\lambda)=w_{1}(1, \lambda) \cos \beta+w_{2}(1, \lambda) \sin \beta
$$

It follows from the formula (6) that

$$
\begin{aligned}
\omega(\lambda)= & \sin (\lambda+\alpha) \cos \beta+\int_{0}^{1}\left[K(1, t) \phi_{0}(t, \lambda)\right]_{1} d t \cos \beta \\
& -\cos (\lambda+\alpha) \sin \beta+\int_{0}^{1}\left[K(1, t) \phi_{0}(t, \lambda)\right]_{2} d t \sin \beta .
\end{aligned}
$$

Integrating by parts we obtain the following asymptotic relations:

$$
\omega(\lambda)=\sin (\lambda+\alpha-\beta)+O\left(\frac{\exp (|\Im \lambda|)}{\lambda}\right) .
$$

The zeros of $\omega(\lambda)$ are the eigenvalues of $L$ and hence it has only simple zeros $\lambda_{n}$.

From this and the estimates for $\omega(\lambda)$ and $H(\lambda)$ it follows that

$$
\chi(\lambda)=\frac{H(\lambda)}{\omega(\lambda)}
$$

is the entire function of order no greater than 1.

It follows from asymptotics (9) and formula (8) that $\chi(\lambda)$ is bounded on any nonreal axis with vertex in origin of $\lambda$-plane. Then it follows from the Phragmen-Lindelöf theorem that $\chi(\lambda)=M$ is constant on the whole $\lambda$-plane.

Let us show that $M=0$. We can rewrite the equation $H(\lambda)=M w(1, \lambda)$ in the form

$$
\begin{aligned}
\int_{0}^{\frac{1}{2}} & p_{1}(x)\left[-\cos 2(\lambda x+\alpha)+\int_{0}^{x} R_{1}(x, t) \exp (2 i \lambda t) d t\right. \\
& \left.+\int_{0}^{x} R_{2}(x, t) \exp (-2 i \lambda t) d t\right] d x+\int_{0}^{\frac{1}{2}} q_{1}(x)[-\sin 2(\lambda x+\alpha) \\
& \left.+\int_{0}^{x} R_{3}(x, t) \exp (2 i \lambda t) d t+\int_{0}^{x} R_{4}(x, t) \exp (-2 i \lambda t) d t\right] d x \\
= & M\left(\sin (\lambda+\alpha-\beta)+O\left(\frac{\exp (|\Im \lambda|)}{\lambda}\right)\right) .
\end{aligned}
$$


By use of the Rimann-Lebesque lemma, the left side of the above equality tends to 0 as $\lambda \rightarrow \infty, \lambda \in R$. Thus we obtained that $M=0$. We are now going to show that $Q(x)=0$ a.e. on $[0,1 / 2]$.

Then we have

$$
\begin{aligned}
& \int_{0}^{\frac{1}{2}} f_{1}(x)\left[\exp (2 i \lambda x)+\int_{0}^{x} S_{11}(x, t) \exp (2 i \lambda t) d t\right. \\
& \left.\quad+\int_{0}^{x} S_{12}(x, t) \exp (-2 i \lambda t) d t\right] d x+\int_{0}^{\frac{1}{2}} f_{2}(x)[\exp (-2 i \lambda x) \\
& \left.\quad+\int_{0}^{x} S_{21}(x, t) \exp (2 i \lambda t) d t+\int_{0}^{x} S_{22}(x, t) \exp (-2 i \lambda t) d t\right] d x=0,
\end{aligned}
$$

where

$$
f_{1}(x)=-\frac{\exp (2 i \alpha)}{2 i}\left(q_{1}(x)+i p_{1}(x)\right), \quad f_{2}(x)=\frac{\exp (-2 i \alpha)}{2 i}\left(q_{1}(x)-i p_{1}(x)\right)
$$

and $S(x, t)=\left(S_{i j}(x, t)\right), i, j=1,2$, is a matrix with entries which are piecewisecontinuously differentiable on $0 \leq t \leq x \leq 1$.

This can be rewritten as

$$
\begin{aligned}
& \int_{0}^{\frac{1}{2}} \exp (2 i \lambda \tau)\left[f_{1}(\tau)+\int_{\tau}^{\frac{1}{2}}\left(f_{1}(x) S_{11}(x, \tau)+f_{2}(x) S_{21}(x, \tau)\right) d x\right] d \tau \\
& +\int_{0}^{\frac{1}{2}} \exp (-2 i \lambda \tau)\left[f_{2}(\tau)+\int_{\tau}^{\frac{1}{2}}\left(f_{1}(x) S_{12}(x, \tau)+f_{2}(x) S_{22}(x, \tau)\right) d x\right] d \tau=0
\end{aligned}
$$

or

$$
\int_{0}^{\frac{1}{2}}\left\langle e_{0}(\lambda \tau), f(\tau)+\int_{\tau}^{\frac{1}{2}} S(x, \tau) f(x) d x\right\rangle d \tau=0 .
$$

Here $e_{0}(x)=(\exp (2 i x), \exp (-2 i x))^{T}$ and $f(x)=\left(f_{1}(x), f_{2}(x)\right)^{T}$. Thus from the completeness of the functions $e_{0}(\lambda \tau)$ in $\left\{L_{2}(0,1 / 2)\right\}^{2}$, it follows that

$$
f(\tau)+\int_{\tau}^{\frac{1}{2}} S(x, \tau) f(x) d x=0 \quad \text { for } \quad 0<\tau<\frac{1}{2} .
$$

But this equation is a homogenous Volterra integral equation and has only the zero solution. Thus we have obtained $f(x)=\left(f_{1}(x), f_{2}(x)\right)^{T}=0$ on $[0,1 / 2]$ and consequently $p_{1}(x)=q_{1}(x)=0$ on $[0,1 / 2]$.

To prove that $p_{1}(x)=q_{1}(x)=0$ on $[1 / 2,1]$, we should repeat arguments for the supplementary problem

$$
l(y)=B y^{\prime}+Q_{1}(x) y, \quad Q_{1}(x)=Q(1-x) \quad(0 \leq x \leq 1)
$$


subject to the boundary conditions

$$
y_{1}(0) \cos \beta+y_{2}(0) \sin \beta=0, \quad y_{1}(1) \cos \alpha+y_{2}(1) \sin \alpha=0 .
$$

\section{§4. Proof of Theorem 2.2}

Let $m(n)$ be a sequence of natural numbers with a property

$$
m(n)=\frac{n}{\sigma}\left(1+\epsilon_{n}\right), \quad 0<\sigma \leq 1, \quad \epsilon_{n} \rightarrow 0 .
$$

We prove an auxiliary lemma

Lemma 1. Let $m(n)$ and $0<b<1 / 2$ are so chosen that $\sigma>2 b$. If for any $n \in Z$

$$
\lambda_{m(n)}=\tilde{\lambda}_{m(n)}, \quad \frac{y_{m(n), 1}(b)}{y_{m(n), 2}(b)}=\frac{\tilde{y}_{m(n), 1}(b)}{\tilde{y}_{m(n), 2}(b)},
$$

then $p(x)=\tilde{p}(x), q(x)=\tilde{q}(x)$ on the $[0, b]$.

Proof. As in the proof of Theorem 2.1 we can show that

$$
\begin{aligned}
G(\lambda) & =\int_{0}^{b}\langle P(x) w(x, \lambda), \tilde{w}(x, \lambda)\rangle d x \\
& =\left.\left(\tilde{w}_{2}(x, \lambda) w_{1}(x, \lambda)-\tilde{w}_{1}(x, \lambda) w_{2}(x, \lambda)\right)\right|_{x=b} .
\end{aligned}
$$

where $P(x)=Q(x)-\tilde{Q}(x)$. Thus from conditions of Theorem 2.2 it follows that

$$
G\left(\lambda_{m(n)}\right)=0, \quad n \in Z .
$$

To prove Lemma, as in the proof of the previous theorem, it is enough to show that $G(\lambda)=0$ on the whole $\lambda$-plane.

From formula (8) and asymptotic (9), it follows that an entire function $G(\lambda)$ is function of exponential type $\leq 2 b$. Let us define an indicator of function $G(\lambda)$ by the formula:

$$
h(\theta)=\limsup _{r \rightarrow \infty} \frac{\ln \left|g\left(r e^{i \theta}\right)\right|}{r} .
$$

Since $|\Im \lambda|=r|\sin \theta|, \theta=\arg \lambda$ it follows that

$$
h(\theta) \leq 2 b|\sin \theta| .
$$


Let us denote by $n(r)$ the number of zeros of $G(\lambda)$ in the disk $|\lambda| \leq r$. There are following asymptotics (see e.g., [6]) for $\lambda_{k}$

$$
\lambda_{n}=\pi n\left(1+O\left(\frac{1}{n}\right)\right) .
$$

These asymptotics and assertions of theorem show that

$$
\lambda_{m(n)}=\frac{\pi n}{\sigma}\left(1+\epsilon_{n}\right), \quad \epsilon_{n} \rightarrow 0 .
$$

Let us now count how many zeroes $\lambda_{n}$ lie inside the disk $\{|\lambda| \leq r\}$. It is easy to see that for a sufficiently large $r$ this quantity is

$$
n(r)=1+2\left[\frac{\sigma r}{\pi}(1+\epsilon(r))\right]=2 \frac{\sigma r}{\pi}(1+\epsilon(r)) .
$$

Here $\epsilon(r) \rightarrow 0$ for $r \rightarrow \infty$ and $[x]$ is the integer part of $x$.

It follows that in the case under consideration

$$
\lim _{r \rightarrow \infty} \frac{n(r)}{r}=\frac{2}{\pi} \sigma>\frac{4 b}{\pi}=\frac{2 b}{\pi} \int_{0}^{2 \pi}|\sin \theta| d \theta \geq \frac{1}{2 \pi} \int_{0}^{2 \pi} h(\theta) d \theta
$$

To complete the proof we have to recall the following theorem [5], p. 173: The set of zeros of every entire function of the exponential type, not identically zero, satisfies the inequality:

$$
\liminf _{r \rightarrow \infty} \frac{n(r)}{r} \leq \frac{1}{2 \pi} \int_{0}^{2 \pi} h(\theta) d \theta .
$$

This theorem and the inequality (12) implies, that $G(\lambda) \equiv 0$ on the whole $\lambda$-plane and, as we already mentioned, it involves $p(x)=\tilde{p}(x), q(x)=\tilde{q}(x)$ on $[0, b]$.

Proof of Theorem 2.2. Firstly let us note that based on the condition

$$
\lambda_{r(n)}=\tilde{\lambda}_{r(n)}, \quad \frac{y_{r(n), 1}(b)}{y_{r(n), 2}(b)}=\frac{\tilde{y}_{r(n), 1}(b)}{\tilde{y}_{r(n), 2}(b)},
$$

it follows from Lemma 1 that $p(x)=\tilde{p}(x), q(x)=\tilde{q}(x)$ a.e. on $[b, 1]$.

Eigenfunctions $y_{n}(x)$ and $\tilde{y}_{n}(x)$ satisfy the same boundary condition on the right end. This means that

$$
w\left(x, \lambda_{n}\right)=C_{n} \tilde{w}\left(x, \lambda_{n}\right)
$$

on $[b, 1]$ for any $n \in Z$ ( $C_{n}$ are constants). Therefore from definition (10) of function $G(\rho)$ it follows that

$$
g\left(\lambda_{n}\right)=0, \quad n \in Z
$$


and, in the same way, that

$$
G\left(\mu_{l(n)}\right)=0, \quad \in Z \text {. }
$$

We are going to show that inequality (12) does not take place and consequently, the entire function of the exponential type $G(\lambda)=0$ on the whole $\lambda$-plane. If we count a quantity of $\lambda_{n}$ and $\mu_{n}$ located inside the disc of radius $r$, we have $1+2[(r / \pi)+O(1)]$ of $\lambda_{n}$ 's and $1+2\left[\left(r \sigma_{1} / \pi\right)+O(1)\right]$ of $\mu_{n}$ 's.

This means that $n(r)=2+2\left[r\left(\sigma_{1}+1\right) / \pi+O(1)\right]$ and

$$
\lim _{r \rightarrow \infty} \frac{n(r)}{r}=\frac{2}{\pi}\left(\sigma_{1}+1\right) .
$$

Repeating the last part of the proof of Lemma we can show that $G(\lambda)=0$ identically on the whole $\lambda$-plane which implies that $p(x)=\tilde{p}(x), q(x)=\tilde{q}(x)$ on $[0, b]$ and, consequently, on $[0,1]$.

\section{References}

[1] Arutyunyan, T. N., Isospectral Dirac operators, Izv. Nats. Akad. Nauk Armenii Mat., 29 (1994), no. 2, 3-14; English transl. in J. Contemp. Math. Anal., Armen. Acad. Sci., 29 (1994), no. 2, 1-10.

[2] Borg, G., Eine Umkehrung der Sturm-Liouvilleschen Eigenwertaufgabe, Bestimmung der Differential gleichung durch die Eigenvert, Acta Math., 78 (1946), 1-96.

[3] Gasymov, M. G. and Levitan, B. M., The Inverse Problem for a Dirac System, Dokl. Akad. Nauk SSSR, 167 (1966), 967-970.

[4] Hochstadt, H. and Lieberman, B., An Inverse Sturm-Liouville Problem with Mixed Given Data, SIAM J. Appl. Math., 34 (1978), 676-680.

[5] Levin, B. Ja., Distribution of zeros of entire functions, AMS Transl. Vol.5, Providence, 1964.

[6] Levitan, B. M. and Sargsjan, I. S., Sturm-Liouville and Dirac Operators, Kluwer Academic Publishers, Dodrecht, Boston, London, 1991.

[7] Malamud, M. M., Uniqueness Questions in Inverse Problems for Systems of Differential Equations on a Finite Interval, Trans. Moscow Math. Soc., 60 (1999), 173-224.

[8] Marchenko, V. A., On certain questions in the theory of differential operators of the second order, Dokl. Akad. Nauk SSSR, 72 (1950), 457-460 (in Russian).

[9] - Some questions in the theory of one-dimensional linear differential operators of the second order. I, Trudy Moscov. Mat. Obsc., 1 (1952), 327-420 (in Russian); Amer. Math. Soc. Transl., Ser. 2101 (1973), 1-104.

[10] Mochizuki, K. and Trooshin, I., Inverse Problem for Interior Spectral Data of SturmLiouville Operator, Seminar Notes of Mathematical Sciences, 3 Ibaraki University, (2000), 44-51.

[11] - Inverse Problem for Interior Spectral Data of Sturm-Liouville Operator, J. Inverse Ill-posed Prob., 9 (2001), 425-433.

[12] Ramm, A. G., Property C for ODE and applications to inverse problems, in Operator theory and applications (Winnipeg, MB, 1998), Fields Inst. Commun., 25, AMS, Providence, RI, (2000), 15-75. 